\title{
Local limit theorems on some non unimodular groups
}

Emile Le Page and Marc Peigné

Abstract. Let $G_{d}$ be the semi-direct product of $\mathbb{R}^{*+}$ and $\mathbb{R}^{d}, d \geq 1$ and let us consider the product group $G_{d, N}=G_{d} \times \mathbb{R}^{N}, N \geq 1$. For a large class of probability measures $\mu$ on $G_{d, N}$, one proves that there exists $\rho(\mu) \in] 0,1]$ such that the sequence of finite measures

$$
\left\{\frac{n^{(N+3) / 2}}{\rho(\mu)^{n}} \mu^{* n}\right\}_{n \geq 1}
$$

converges weakly to a non-degenerate measure.

Résumé. Soit $G_{d}$ le produit semi-direct de $\mathbb{R}^{*+}$ et de $\mathbb{R}^{d}$ et $G_{d, N}$ le groupe produit $G_{d} \times \mathbb{R}^{N}, N \geq 0$. Pour une large classe de mesures de probabilité sur $G_{d, N}$ nous montrons qu'il existe $\left.\left.\rho(\mu) \in\right] 0,1\right]$ tel que la suite de mesures finies

$$
\left\{\frac{n^{(N+3) / 2}}{\rho(\mu)^{n}} \mu^{* n}\right\}_{n \geq 1}
$$

converge vaguement vers une mesure non nulle.

\section{Introduction.}

Fix two integers $d \geq 1, N \geq 0$ and choose a norm $\|\cdot\|$ on $\mathbb{R}^{d}$ and $\mathbb{R}^{N}$ (when $N \geq 1$ ). Let $G_{d, N}$ be the connected group $\mathbb{R}^{*+} \times \mathbb{R}^{d} \times \mathbb{R}^{N}$ 
with the composition law

$$
\begin{aligned}
& \text { for all } g=(a, u, b), \text { for all } g^{\prime}=\left(a^{\prime}, u^{\prime}, b^{\prime}\right) \in G, \\
& g \cdot g^{\prime}=\left(a a^{\prime}, a u^{\prime}+u, b+b^{\prime}\right) .
\end{aligned}
$$

We will note $g=(a(g), u(g), b(g))$ (or $g=(a, u, b)$ when there is no ambiguity). The group $\left(G_{d, N}, \cdot\right)$ is a non unimodular solvable group with exponential growth and the right Haar measure $m_{D}$ on $G_{d, N}$ is

$$
m_{D}\left(d a d u d b_{1} \cdots d b_{N}\right)=\frac{d a d u d b_{1} \cdots d b_{N}}{a} .
$$

Note that $G_{d, 0}$ is the semi-direct product of $\mathbb{R}^{*+}$ and $\mathbb{R}^{d}$; in particular $G_{1,0}$ is the affine group of the real line.

We consider a probability measure $\mu$ on $G$; we denote by $\mu^{* n}$ its $n^{\text {th }}$ power of convolution. Under quite general assumptions on $\mu$ we show that there exists $\rho(\mu) \in] 0,1[$ such that the sequence

$$
\left\{\frac{n^{(N+3) / 2}}{\rho(\mu)^{n}} \mu^{* n}\right\}_{n \geq 0}
$$

converges weakly to a non-degenerate measure. This problem has already been tackled by $\mathrm{Ph}$. Bougerol in [3] where were established local limit theorems on some solvable groups with exponential growth; in particular, for a class $R$ of probability measures $\mu$ on the affine group of the real line (that is $d=1$ and $N=0$ ) he showed that the sequence

$$
\left\{\frac{n^{3 / 2}}{\rho(\mu)^{n}} \mu^{* n}\right\}_{n \geq 0}
$$

converges weakly to a non-degenerate measure. In [7] we extend this result to a quite large class of probability measures; the new ingredient in our proof was the fact that there exists closed connections between this problem and the theory of the fluctuations of a random walk on the real line. In the present paper, we extend this result to the case $N \geq 1$; we first obtain uniform upperbounds in the Local limit theorem for a random walk on $\mathbb{R}^{d}$ and, secondly, we use a generalisation of the Wiener-Hopf's factorisation due to Ch. Sunyach [9].

This study is also related with the work by N. T. Varopoulos [10], [11] where upperbounds and lowerbounds for the asymptotic behaviour 
of the convolution powers $\mu^{* n}$ of a large class of probability measures are given.

From now on, we will suppose that $N \geq 1$ and we set $G=G_{d, N}$. We introduce the following conditions on $\mu$ :

Hypothesis G1. There exists $\alpha>0$ such that

$$
\int_{G}\left(e^{\alpha|\log a|}+\|u\|^{\alpha}+\|b\|^{2}\right) \mu(d a d u d b)<+\infty .
$$

Hypothesis G2. $\int_{G} \log a \mu(d a d u d b)=0$ and $\int_{G} b \mu(d a d u d b)=0$.

Hypothesis G3. The support of $\mu$ is included in $\mathbb{R}^{*+} \times\left(\mathbb{R}^{+}\right)^{d} \times \mathbb{R}^{N}$, the image of $\mu$ by the mapping $(a, u, b) \longmapsto(\log a, b)$ is aperiodic in $\mathbb{R}^{N+1}$ (see Definition 2.1) and there exists $\beta>0$ such that

$$
\int_{G}\|u\|^{-\beta} \mu(d a d u d b)<+\infty .
$$

Hypothesis G'3. The measure $\mu$ is absolutely continuous with respect to the Haar measure $m_{D}$ on $G$ and its density $\phi_{\mu}$ satisfies

$$
\int_{] 0,1] \times \mathbb{R}^{N}} \sqrt[q]{\int_{\mathbb{R}} \phi_{\mu}^{q}(a, u, b) d u} \frac{d a d b}{a^{\gamma}}<+\infty .
$$

for some $\gamma$ and $q$ in $] 1,+\infty[$.

We have the

Theorem 1.1. Let $\mu$ be a probability measure on $G$ satisfying hypotheses G1, G2 and G3 (or G'3). Then, the sequence of finite measures $\left\{n^{(N+3) / 2} \mu^{* n}\right\}_{n \geq 0}$ converges weakly to a non-degenerate Radon measure on $G$.

Note that the asymptotic behavior of the sequence $\left\{\mu^{* n}\right\}_{n \geq 1}$ does not depend on $d$.

When $\mu$ is not centered, that is

$$
\int_{G} \log a \mu(d a d u d b) \neq 0
$$


or

$$
\int_{G} b \mu(d a d u d b) \neq 0,
$$

we bring back the study to the centered case as in [7]. We introduce the following conditions on $\mu$ :

Hypothesis $\mathbf{G}^{*} \mathbf{1}$. There exists $\alpha>0$ such that

$$
\int_{G}\left(a^{t}+\|u\|^{\alpha}+\exp (t\|b\|)\right) \mu(d a d u d b)<+\infty
$$

for any $t \in \mathbb{R}$.

Hypothesis $\mathbf{G}^{*}$ 2. One has

$$
\int_{G} \log a \mu(d a d u d b) \neq 0
$$

with $\mu\{g \in G: a(g)<1\}>0$ and $\mu\{g \in G: a(g)>1\}>0$.

When $\mu$ satisfies these two conditions, there exists a unique $\left(s_{0}, t_{0}\right)$ $\in \mathbb{R} \times \mathbb{R}^{N}$ such that

$$
\int_{G} a^{s_{0}} e^{\left\langle t_{0}, b\right\rangle} \mu(d a d u d b)=\inf _{(s, t) \in \mathbb{R} \times \mathbb{R}^{N}} \int_{G} a^{s} e^{\langle t, b\rangle} \mu(d a d u d b) .
$$

Furthermore,

$$
\rho(\mu)=\int_{G} a^{s_{0}} e^{\left\langle t_{0}, b\right\rangle} \mu(d a d u d b)
$$

belongs to $] 0,1]$. Note that the probability measure

$$
\mu_{0}(d g)=\frac{1}{\rho(\mu)} a(g)^{s_{0}} e^{\left\langle t_{0}, b(g)\right\rangle} \mu(d g)
$$

satisfies hypotheses G1 and G2. The following condition is the equivalent of Hypothesis G'3 in the non centered case:

Hypothesis $\mathbf{G}^{*} 3$. The measure $\mu$ is absolutely continuous with respect to the Haar measure $m_{D}$ on $G$ and its density $\phi_{\mu}$ satisfies

$$
\int_{] 0,1] \times \mathbb{R}^{N}} \sqrt[q]{\int_{\mathbb{R}} \phi_{\mu}^{q}(a, u, b) d u} \frac{d a d b}{a^{\gamma}}<+\infty
$$


for some $q \in] 1,+\infty[$ and $\gamma \in] 1-s_{0},+\infty[$.

Theorem 1.2. Let $\mu$ be a probability measure on $G$ satisfying conditions $\mathrm{G}^{*} 1, \mathrm{G}^{*} 2$ and $\mathrm{G} 3$ (or $\mathrm{G}^{*} 3$ ) and let

$$
\rho(\mu)=\inf _{(s, t) \in \mathbb{R} \times \mathbb{R}^{N}} \int_{G} a^{s} e^{\langle t, b\rangle} \mu(d a d u d b) .
$$

Then, the sequence of finite measures

$$
\left\{\frac{n^{(N+3) / 2}}{\rho(\mu)^{n}} \mu^{* n}\right\}_{n \geq 1}
$$

weakly converges to a non-degenerate Radon measure on $G$.

The demonstration of Theorem 2.1 is closely related to the study of the fluctuations of a random walk $\left(X_{1}^{n}, Y_{1}^{n}\right)_{n \geq 0}$ on $\mathbb{R}^{N+1}$. In Section 2 , we first state the classical local limit theorem on $\mathbb{R}^{N+1}$ but we add in its statement uniform upperbounds relatively to the starting point of the random walk $\left(X_{1}^{n}, Y_{1}^{n}\right)_{n \geq 0}$. This result is thus very usefull to obtain a precise equivalent in Theorem 2.5 of the joint law of the random walk $\left(X_{1}^{n}, Y_{1}^{n}\right)_{n \geq 0}$ with its first entrance time $T_{+}$in the half space $\mathbb{R}^{+} \times \mathbb{R}^{N}$; a local limit theorem for the process

$$
\left(X_{1}^{n}, \max \left\{0, X_{1}^{1}, \ldots, X_{1}^{n}\right\}, Y_{1}^{n}\right)_{n \geq 0}
$$

is thus obtained (Theorem 2.6). In Section 3 we give the main steps of the proof of Theorem 1.1.

\section{Fluctuations of a random walk on $\mathbb{R}^{N+1}$.}

Fix an integer $N \geq 1$ and let $\left(X_{1}, Y_{1}\right),\left(X_{2}, Y_{2}\right), \ldots$ be independent $\mathbb{R} \times \mathbb{R}^{N}$-valued random variables with distribution $p$ defined on a probability space $(\Omega, \mathcal{F}, \mathbb{P})$. Let $\left(X_{1}^{n}, Y_{1}^{n}\right)_{n \geq 0}$ be the associated random walk on $\mathbb{R} \times \mathbb{R}^{N}$ starting from $(0,0)$ and defined by $X_{1}^{0}=0, Y_{1}^{0}=0$ and $X_{1}^{n}=X_{1}+\cdots+X_{n}, Y_{1}^{n}=Y_{1}+\cdots+Y_{n}$ for $n \geq 1$; the distribution of the couple $\left(X_{1}^{n}, Y_{1}^{n}\right)$ is the $n^{\text {th }}$ power of convolution $p^{* n}$ of the measure $p$. Denote by $\mathcal{F}_{n}$ the $\sigma$-algebra generated by $\left(X_{1}, Y_{1}\right), \ldots,\left(X_{n}, Y_{n}\right), n \geq 1$.

Let us first recall the 
Definition 2.1. Let $p$ be a probability measure on $\mathbb{R}^{k}, k \geq 1$. The measure $p$ is aperiodic on $\mathbb{R}^{k}$ if there is no closed and proper subgroup $H$ of $\mathbb{R}^{k}$ and no $\alpha \in \mathbb{R}^{k}$ such that $p(\alpha+H)=1$.

Denote by $\hat{p}$ the characteristic function of $p$ defined by $\hat{p}(u, v)=$ $\mathbb{E}\left[e^{i u X_{1}+i\left\langle v, Y_{1}\right\rangle}\right]$ for any $(u, v) \in \mathbb{R} \times \mathbb{R}^{N}$. Recall that the probability measure $p$ is aperiodic if and only if $|\hat{p}(u, v)|<1$ for $(u, v) \neq(0,0)$.

For any $\mathcal{A} \subset \mathbb{R} \times \mathbb{R}^{N}$ let $\left\{T_{\mathcal{A}}^{(k)}\right\}_{k \geq 0}$ be the the successive times of visit of the random walk $\left(X_{1}^{n}, Y_{1}^{n}\right)_{n \geq 1}$ to the set $\mathcal{A}$; one has $T_{\mathcal{A}}^{(0)}=$ $0, T_{\mathcal{A}}^{(1)}=\inf \left\{n \geq 1:\left(X_{1}^{n}, Y_{1}^{n}\right) \in \mathcal{A}\right\}$ and $T_{\mathcal{A}}^{(k+1)}=\inf \left\{n \geq T_{\mathcal{A}}^{(k)}+1:\right.$ $\left.\left(X_{1}^{n}, Y_{1}^{n}\right) \in \mathcal{A}\right\}$. Note that the $T_{\mathcal{A}}^{(k)}$ are stopping times with respect to the filtration $\left\{\mathcal{F}_{n}\right\}_{n \geq 1}$. We will associate to $(p, \mathcal{A})$ the transition kernel $P_{\mathcal{A}}$ defined by

$$
P_{\mathcal{A}}((x, y), \mathcal{B})=\int_{\mathbb{R} \times \mathbb{R}^{N}} \mathbf{1}_{\mathcal{A}^{c} \cap \mathcal{B}}\left(x+x^{\prime}, y+y^{\prime}\right) p\left(d x^{\prime} d y^{\prime}\right),
$$

for any Borel set $\mathcal{B}$ in $\mathbb{R} \times \mathbb{R}^{N}$; note that for any $k \geq 1$ one has $P_{\mathcal{A}}^{k}((0,0), \mathcal{B})=\mathbb{E}\left[\left[T_{\mathcal{A}}>k\right] ;\left(X_{1}^{k}, Y_{1}^{k}\right) \in \mathcal{B}\right]$. In order to simplify the notations we will set $T_{-}=T_{\mathbb{R}^{-} \times \mathbb{R}^{N}}, P_{-}=P_{\mathbb{R}^{-} \times \mathbb{R}^{N}}$ and $T_{-}^{(k)}=T_{\mathbb{R}^{-} \times \mathbb{R}^{N}}^{(k)}$; similar notations will hold, with obvious modifications, when $\mathcal{A}=$ $\mathbb{R}^{*-} \times \mathbb{R}^{N}, \mathbb{R}^{+} \times \mathbb{R}^{N}$ and $\mathbb{R}^{*+} \times \mathbb{R}^{N}$.

Troughout this paragraph, for any $k \geq 1$, we denote by $\lambda_{k}$ the Lebesgue measure on $\mathbb{R}^{k}$. Furthermore, for any $\delta>0, \mathcal{H}_{\delta}\left(\mathbb{R}^{k}\right)$ is the space of $\mathbb{C}$-valued functions $\varphi$ on $\mathbb{R}^{k}$ such that

$$
\sup _{x \in \mathbb{R}^{k}}\left(1+\|x\|^{\delta}\right)^{k}|\varphi(x)|<+\infty .
$$

\subsection{Preliminaries.}

The local limit theorem gives the asymptotic behaviour of the sequence $\left\{p^{* n}(\varphi)\right\}_{n \geq 1}$ for any continuous function $\varphi$ with compact support on $\mathbb{R}^{N+1}$; we state it here and we precise some uniform upperbound for the sequence $\left\{p^{* n}(\varphi)\right\}_{n \geq 1}$ when $\varphi$ belongs to $\mathcal{H}_{\delta}\left(\mathbb{R}^{N+1}\right)$ with $\delta>4$.

Theorem 2.2. Assume that:

i) the common distribution $p$ of the variables $\left(X_{n}, Y_{n}\right), n \geq 1$, is aperiodic on $\mathbb{R}^{N+1}$, 
ii) $\mathbb{E}\left[\left|X_{1}\right|^{2}+\left\|Y_{1}\right\|^{2}\right]<+\infty$ and $\mathbb{E}\left[X_{1}\right]=0, \mathbb{E}\left[Y_{1}\right]=0$.

Then:

i) for any continuous function $\varphi$ with compact support on $\mathbb{R}^{N+1}$ one has

$$
\begin{array}{rl}
\lim _{n \rightarrow+\infty} n^{(N+1) / 2} & \mathbb{E}\left[\varphi\left(X_{1}^{n}, Y_{1}^{n}\right)\right] \\
& =\frac{1}{(2 \pi)^{(N+1) / 2} \sqrt{|C|}} \int_{\mathbb{R}^{N+1}} \varphi(x, y) \lambda_{1}(d x) \lambda_{N}(d y),
\end{array}
$$

where $|C|$ denotes the determinant of the positive definite quadratic form

$$
C(u, v)=\mathbb{E}\left[\left(u X_{1}+\left\langle v, Y_{1}\right\rangle\right)^{2}\right] .
$$

ii) For any function $\varphi$ in $\mathcal{H}_{\delta}\left(\mathbb{R}^{N+1}\right)$ with $\delta>4$, the sequence $\left\{n^{(N+1) / 2} \mathbb{E}\left[\varphi\left(x+X_{1}^{n}, y+Y_{1}^{n}\right)\right]\right\}_{n \geq 1}$ is bounded uniformly in $(x, y) \in$ $\mathbb{R} \times \mathbb{R}^{N}$.

Proof. The first assumption is the classical local limit theorem. To obtain the second claim, fix a non negative function $\phi$ whose Fourier transform has a compact support $K(\hat{\phi})$. Recall that

$$
\hat{p}(u, v)=1-\frac{1}{2} C(u, v)(1+\varepsilon(u, v))
$$

with $\lim _{(u, v) \rightarrow(0,0)} \varepsilon(u, v)=0$; so there exists $\delta>0$ such that for $|u|+$ $\|v\|<\delta$ one has

$$
|\hat{p}(u, v)| \leq 1-\frac{1}{4} C(u, v) \leq e^{-C(u, v) / 4}
$$

On the other hand, by the aperiodicity of $p$ there exists $\rho=\rho(p, K(\hat{\phi}))$ such that $|\hat{p}(u, v)| \leq \rho$ as soon as $(u, v)$ belongs to $K(\hat{\phi})$ and $|u|+\|v\| \geq$ $\delta$. It follows that

$$
\begin{aligned}
& (2 \pi n)^{(N+1) / 2} \mathbb{E}\left[\phi\left(X_{1}^{n}, Y_{1}^{n}\right)\right] \\
& \leq n^{(N+1) / 2} \int_{|u|+\|v\|<\delta}|\hat{\phi}(u, v)||\hat{p}(u, v)|^{n} \lambda_{1}(d u) \lambda_{N}(d v) \\
& \quad+n^{(N+1) / 2} \int_{|u|+\|v\| \geq \delta}|\hat{\phi}(u, v)||\hat{p}(u, v)|^{n} \lambda_{1}(d u) \lambda_{N}(d v)
\end{aligned}
$$




$$
\begin{aligned}
& \leq n^{(N+1) / 2} \int_{|u|+\|v\|<\delta n^{(N+1) / 2}}\left|\hat{\phi}\left(\frac{u}{\sqrt{n}}, \frac{v}{\sqrt{n}}\right)\right| e^{-(n / 4) C(u / \sqrt{n}, v / \sqrt{n})} \\
& \quad \cdot \lambda_{1}(d u) \lambda_{N}(d v) \\
& +n^{(N+1) / 2} \rho^{n}\|\hat{\phi}\|_{1} \\
& \leq\|\hat{\phi}\|_{\infty} \int_{\mathbb{R} \times \mathbb{R}^{N}} e^{-C(u, v) / 4} \lambda_{1}(d u) \lambda_{N}(d v)+n^{(N+1) / 2} \rho^{n}\|\hat{\phi}\|_{1} .
\end{aligned}
$$

Now set $\phi_{x, y}\left(x^{\prime}, y^{\prime}\right)=\phi\left(x+x^{\prime}, y+y^{\prime}\right)$ for any $(x, y) \in \mathbb{R} \times \mathbb{R}^{N}$ and note that $\hat{\phi}_{x, y}(u, v)=e^{i u x+i\langle v, y\rangle} \hat{\phi}(u, v)$; the functions $\hat{\phi}_{x, y}$ and $\hat{\phi}$ thus have the same compact support and satisfies the equalities $\left\|\hat{\phi}_{x, y}\right\|_{1}=\|\hat{\phi}\|_{1}$ and $\left\|\hat{\phi}_{x, y}\right\|_{\infty}=\|\hat{\phi}\|_{\infty}$. For any $(x, y) \in \mathbb{R} \times \mathbb{R}^{N}$ one thus has

$$
\begin{aligned}
& \left|(2 \pi n)^{(N+1) / 2} \mathbb{E}\left[\phi_{x, y}\left(X_{1}^{n}, Y_{1}^{n}\right)\right]\right| \\
& \quad \leq\|\hat{\phi}\|_{\infty} \int_{\mathbb{R} \times \mathbb{R}^{N}} e^{-C(u, v) / 4} \lambda_{1}(d u) \lambda_{N}(d v)+n^{(N+1) / 2} \rho^{n}\|\hat{\phi}\|_{1} .
\end{aligned}
$$

The assertion ii) thus holds for any function $\phi$ whose Fourier transform has a compact support. To achieve the proof of ii) it suffices to show that for any function $\varphi$ in $\mathcal{H}_{\delta}\left(\mathbb{R}^{N+1}\right)$ with $\delta>4$ there exists a function $\phi$ whose Fourier transform has a compact support and $|\varphi| \leq \phi$. It is an immediate consequence of the following result; we thank here J. P. Conze for helpfull discussions about this fact.

Lemma 2.3. Set

$$
h_{\varepsilon}(x)=\frac{1}{1+|x|^{4+\varepsilon}}
$$

for any $x \in \mathbb{R}$. If $\varepsilon>0$ there exists a function $\overline{h_{\varepsilon}}$ greater than $h_{\varepsilon}$ and whose Fourier transform has a compact support in $\mathbb{R}$.

Proof. Set

$$
\overline{h_{\varepsilon}}(x)=C\left(\frac{\sin ^{2} x}{x^{2}}+\frac{\sin ^{2} \alpha x}{x^{2}}\right)
$$

for some $\alpha$ and $C$ in $\mathbb{R}^{*+}$ which will depend on $\varepsilon$. Assume $\alpha \notin \mathbb{Q}$, the function $\overline{h_{\varepsilon}}$ is strictly positive on $\mathbb{R}$; it thus suffices to show that there exists $\alpha \notin \mathbb{Q}$ such that

$$
\lim _{x \rightarrow+\infty} x^{2+\varepsilon}\left(\sin ^{2} x+\sin ^{2}(\alpha x)\right)=+\infty
$$


If such a real did not exist, then for any $\alpha \notin \mathbb{Q}$ there should exist a sequence $\left\{x_{n}\right\}_{n \geq 1}$ which tends to $+\infty$ and a constant $C_{\varepsilon}>0$ such that for all $n \geq 1$,

$$
\sin ^{2} x_{n}+\sin ^{2}\left(\alpha x_{n}\right) \leq \frac{C}{x_{n}^{2+\varepsilon}} .
$$

So there should exist two strictly increasing sequences of integers $\left\{k_{n}\right\}_{n \geq 1}$ and $\left\{l_{n}\right\}_{n \geq 1}$ such that

$$
\left|x_{n}-k_{n} \pi\right| \leq \frac{C^{\prime}}{x_{n}^{1+\varepsilon / 2}}, \quad\left|\alpha x_{n}-l_{n} \pi\right| \leq \frac{C^{\prime}}{x_{n}^{1+\varepsilon / 2}}
$$

which implies

$$
\left|\alpha-\frac{l_{n}}{k_{n}}\right| \leq \frac{C^{\prime \prime}}{k_{n}^{2+\varepsilon / 2}}
$$

for some positive constants $C^{\prime}$ and $C^{\prime \prime}$. This leads to a contradiction because for almost all $\alpha \in \mathbb{R}$ (with respect with the Lebesgue measure), this last inequality has at most a finite number of solutions in $\mathbb{N}^{2}[2]$. The lemma is proved.

\subsection{A local limit theorem for a killed random walk on a half space.}

In [7], we proved the following

Theorem 2.4. Let the hypotheses of Theorem 2.2 hold. Then for any continuous function with compact support $\varphi$ on $\mathbb{R}^{-}$we have

$$
\lim _{n \rightarrow+\infty} n^{3 / 2} \mathbb{E}\left[\left[T_{+}>n\right] ; \varphi\left(X_{1}^{n}\right)\right]=\frac{1}{\sigma\left(X_{1}\right) \sqrt{2 \pi}} \int_{-\infty}^{0} \varphi(x) \lambda_{1}^{-} * U^{*-}(d x),
$$

where $\lambda_{1}^{-}$denotes the restriction of the Lebesgue measure on $\mathbb{R}^{-}$and $U^{*-}$ is the $\sigma$-finite measure on $\mathbb{R}^{-}$defined by

$$
U^{*-}(\mathcal{B})=\sum_{k=1}^{+\infty} \mathbb{E}\left[\mathbf{1}_{\mathcal{B}}\left(X_{1}^{T^{(k)}}\right)\right]
$$

for any Borel set $\mathcal{B}$. In the same way, one has

$$
\lim _{n \rightarrow+\infty} n^{3 / 2} \mathbb{E}\left[\left[T_{*+}>n\right] ; \varphi\left(X_{1}^{n}\right)\right]=\frac{1}{\sigma\left(X_{1}\right) \sqrt{2 \pi}} \int_{-\infty}^{0} \varphi(x) \lambda_{1}^{-} * U^{-}(d x),
$$


where $U^{-}$is the $\sigma$-finite measure on $\mathbb{R}^{-}$defined by

$$
U^{-}(\mathcal{B})=\sum_{k=1}^{+\infty} \mathbb{E}\left[\mathbf{1}_{\mathcal{B}}\left(X_{1}^{T_{-}^{(k)}}\right)\right]
$$

for any Borel set $\mathcal{B}$.

Recall that the random walks $\left\{X_{1}^{T_{-}^{(k)}}\right\}_{k \geq 1}$ and $\left\{X_{1}^{T_{*-}^{(k)}}\right\}_{k \geq 1}$ are transient on $\mathbb{R}^{-} ;$it follows that the series $\sum_{k=0}^{+\infty} \mathbb{E}\left[\left[T_{+}>k\right] ; \varphi\left(x+X_{1}^{k}\right)\right]$ and $\sum_{k=0}^{+\infty} \mathbb{E}\left[\left[T_{*+}>k\right] ; \varphi\left(x+X_{1}^{k}\right)\right]$ do converge. Furthermore one has

$$
\sum_{k=0}^{+\infty} \mathbb{E}\left[\left[T_{+}>k\right] ; \varphi\left(x+X_{1}^{k}\right)\right]=\int_{-\infty}^{0} \varphi(x) U^{*-}(d x)
$$

and

$$
\sum_{k=0}^{+\infty} \mathbb{E}\left[\left[T_{*+}>k\right] ; \varphi\left(x+X_{1}^{k}\right)\right]=\int_{-\infty}^{0} \varphi(x) U^{-}(d x) .
$$

Let us now state the following

Theorem 2.5. Let the hypotheses of Theorem 2.2 hold. Then:

i) For any continuous function $\varphi$ with compact support on $\mathbb{R}^{-} \times \mathbb{R}^{N}$ one has

$$
\begin{aligned}
\lim _{n \rightarrow+\infty} & \left.n^{(N+3) / 2} \mathbb{E}\left[\left[T_{+}>n\right] ; \varphi\left(X_{1}^{n}, Y_{1}^{n}\right)\right)\right] \\
& =\frac{1}{(2 \pi)^{(N+1) / 2} \sqrt{|C|}} \int_{\mathbb{R}^{-} \times \mathbb{R}^{N}} \varphi(x, y) \lambda_{1}^{-} * U^{*-}(d x) \lambda_{N}(d y) .
\end{aligned}
$$

ii) For any continuous function $f$ with compact support on $\mathbb{R}$ and any $g$ in $\mathcal{H}_{\delta}\left(\mathbb{R}^{N}\right)$ with $\delta>4$, the sequence

$$
\left\{n^{(N+3) / 2} \mathbb{E}\left[\left[T_{+}>n\right] ; f\left(X_{1}^{n}\right) g\left(y+Y_{1}^{n}\right)\right]\right\}_{n \geq 1}
$$

is bounded, uniformly in $y \in \mathbb{R}^{N}$.

In the same way, one has

$$
\begin{aligned}
\lim _{n \rightarrow+\infty} n^{(N+3) / 2} \mathbb{E}\left[\left[T_{*+}>n\right] ; \varphi\left(X_{1}^{n}, Y_{1}^{n}\right)\right] \\
\quad=\frac{1}{(2 \pi)^{(N+1) / 2} \sqrt{|C|}} \int_{\mathbb{R}^{-} \times \mathbb{R}^{N}} \varphi(x, y) \lambda_{1}^{-} * U^{-}(d x) \lambda_{N}(d y)
\end{aligned}
$$


and the sequence

$$
\left\{n^{(N+3) / 2} \mathbb{E}\left[\left[T_{+}>n\right] ; f\left(X_{1}^{n}\right) g\left(y+Y_{1}^{n}\right)\right]\right\}_{n \geq 1}
$$

is bounded, uniformly in $y \in \mathbb{R}^{N}$.

Proof. We prove this theorem by induction over $N$. Theorem 2.2 deals with the case $N=0$; we will suppose that this result hold for some $N \geq$ 0 and we consider a sequence $\left(X_{n}, Y_{n}, Z_{n}\right)_{n \geq 1}$ of independent identically distributed random variables on $\mathbb{R} \times \mathbb{R}^{N} \times \mathbb{R}$. By a classical argument in probability theory, it suffices to show the above convergence hold for $\varphi(x, y, z)=e^{a x} \mathbf{1}_{\mathbb{R}^{-}}(x) \phi(y) \psi(z)$ where $a \in \mathbb{R}^{*+}$ and $\phi, \psi$ are $\mathbb{C}$ valued functions whose Fourier transform are continuous with compact supports. By the inverse Fourier transform one has

$$
\begin{aligned}
I_{n} & =\mathbb{E}\left[\left[T_{+}>n\right] ; e^{a X_{1}^{n}} \phi\left(Y_{1}^{n}\right) \psi\left(Z_{1}^{n}\right)\right] \\
& =\frac{1}{(2 \pi)^{(N+1) / 2}} \int_{\mathbb{R}^{N} \times \mathbb{R}} \hat{\phi}(v) \hat{\psi}(w) \alpha_{n}(a, v, w) \lambda_{N}(d v) \lambda_{1}(d w)
\end{aligned}
$$

with $\alpha_{n}(a, v, w)=\mathbb{E}\left[\left[T_{+}>n\right] ; e^{a X_{1}^{n}+i\left\langle v, Y_{1}^{n}\right\rangle+i w Z_{1}^{n}}\right]$.

The Spitzer's factorisation for random walks on $\mathbb{R}$ gives for all $a>0$, for all $s \in[0,1[$

$$
\sum_{n=0}^{+\infty} s^{n} \mathbb{E}\left[\left[T_{+}>n\right] ; e^{a X_{1}^{n}}\right]=\exp \left(\sum_{n=1}^{+\infty} \frac{s^{n}}{n} \mathbb{E}\left[\left[X_{1}^{n}<0\right] ; e^{a X_{1}^{n}}\right]\right)
$$

Using the fact that $\mathbb{R}^{+} \times \mathbb{R}^{N+1}$ and $\mathbb{R}^{*-} \times \mathbb{R}^{N+1}$ are semi-groups in $\mathbb{R}^{N+2}$, Ch. Sunyach extended this factorisation to the multidimensionnal case ([9, Corollary 3, p. 553 and Theorem 5, p. 556]); for any $a>0$, $v \in \mathbb{R}^{N}, w \in \mathbb{R}$ and $s \in[0,1[$ one thus has

$$
\begin{aligned}
& \sum_{n=0}^{+\infty} s^{n} \mathbb{E}\left[\left[T_{+}>n\right] ; e^{a X_{1}^{n}+i\left\langle v, Y_{1}^{n}\right\rangle+i w Z_{1}^{n}}\right] \\
&=\exp \left(\sum_{n=1}^{+\infty} \frac{s^{n}}{n} \mathbb{E}\left[\left[X_{1}^{n}<0\right] ; e^{a X_{1}^{n}+i\left\langle v, Y_{1}^{n}\right\rangle+i w Z_{1}^{n}}\right]\right)
\end{aligned}
$$

that is

$$
(n+1) \alpha_{n+1}(a, v, w)=\sum_{k=0}^{n} \beta_{n+1-k}(a, v, w) \alpha_{k}(a, v, w)
$$


with $\beta_{n}(a, v, w)=\mathbb{E}\left[\left[X_{1}^{n}<0\right] ; e^{a X_{1}^{n}+i\left\langle v, Y_{1}^{n}\right\rangle+i w Z_{1}^{n}}\right]$. Finally

$$
I_{n}=\frac{1}{n+1} \sum_{k=0}^{n} I_{n, k}
$$

with

$$
\begin{array}{r}
I_{n, k}=\frac{1}{(2 \pi)^{(N+1) / 2}} \int_{\mathbb{R}^{N} \times \mathbb{R}} \beta_{n+1-k}(a, v, w) \alpha_{k}(a, v, w) \\
\cdot \hat{\phi}(v) \hat{\psi}(w) \lambda_{N}(d v) \lambda_{1}(d w) .
\end{array}
$$

Set

$$
\begin{aligned}
I=\frac{1}{(2 \pi)^{(N+2) / 2} \sqrt{|C|}} \int_{\mathbb{R}^{N} \times \mathbb{R}} \sum_{k=0}^{+\infty} \mathbb{E}\left[\left[T_{+}>k\right] ; \frac{e^{a X_{1}^{k}}}{a}\right] \\
\cdot \phi(y) \psi(z) \lambda_{N}(d y) \lambda_{1}(d z),
\end{aligned}
$$

since

$$
I=\lambda_{1}^{-} * U^{*-}\left(e^{a \cdot}\right) \lambda_{N}(\phi) \lambda_{1}(\psi),
$$

it suffices to show that $\left\{n^{(N+4) / 2} I_{n}\right\}_{n \geq 1}$ converges to $I$, that is

1) for all $k>0, \lim _{n \rightarrow+\infty} n^{(N+2) / 2} I_{n, k}=I_{* k}$,

2) $\sum_{k=0}^{+\infty}\left|I_{* k}\right|<+\infty$ and $\sum_{k=0}^{+\infty} I_{* k}=I$,

3) $\limsup _{l \rightarrow+\infty} \limsup _{n \rightarrow+\infty} n^{(N+2) / 2} \sum_{k=l}^{n}\left|I_{n, k}\right|=0$.

To prove the assertion 1 ), note that

$$
I_{n, k}=\mathbb{E}\left[\left[T_{+}>k\right] \cap\left[X_{k+1}^{n+1}>0\right] ; e^{a X_{1}^{n+1}} \phi\left(Y_{1}^{n+1}\right) \psi\left(Z_{1}^{n+1}\right)\right],
$$

by the local limit theorem on $\mathbb{R}^{N+2}$ the assertion 1 ) follows with

$$
\begin{aligned}
I_{* k}= & \frac{1}{2 \pi^{(N+2) / 2} \sqrt{|C|}} \frac{\mathbb{E}\left[\left[T_{+}>k\right] ; e^{a X_{1}^{k}}\right]}{a} \\
& \cdot \int_{\mathbb{R}^{N}} \phi(y) \lambda_{N}(d y) \int_{\mathbb{R}} \psi(z) \lambda_{1}(d z) .
\end{aligned}
$$


The fact that the series $\sum_{k=0}^{+\infty}\left|I_{* k}\right|$ converges is a direct consequence of Theorem 2.4. To prove the assertion 3), note that

$$
\begin{aligned}
\left|I_{n, k}\right| & \leq \mathbb{E}\left[\left[T_{+}>k\right] \cap\left[X_{k+1}^{n+1}<0\right] ; e^{a X_{1}^{n+1}}\left|\phi\left(Y_{1}^{n+1}\right)\right|\left|\psi\left(Z_{1}^{n+1}\right)\right|\right] \\
& \leq \mathbb{E}\left[\left[T_{+}>k\right] ; e^{a X_{1}^{k}} \int_{\mathbb{R}^{-} \times \mathbb{R}^{N} \times \mathbb{R}} e^{a x}\left|\phi\left(y+Y_{1}^{k}\right)\right|\right. \\
& \left.\cdot\left|\psi\left(z+Z_{1}^{k}\right)\right| p^{*(n+1-k)}(d x d y d z)\right] \\
& \leq \frac{C(a, \phi, \psi)}{(n+1-k)^{(N+2) / 2}} \mathbb{E}\left[\left[T_{+}>k\right] ; e^{a X_{1}^{k}}\right] \quad \text { by Theorem 2.2.ii) } \\
& \leq \frac{C_{1}}{(n+1-k)^{(N+2) / 2} k^{3 / 2}} \quad \text { by Theorem 2.4. }
\end{aligned}
$$

On the other hand

$$
\begin{aligned}
\left|I_{n, k}\right| \leq & \|\psi\|_{\infty} \int_{\mathbb{R}^{-} \times \mathbb{R}^{N} \times \mathbb{R}} \mathbb{E}\left[\left[T_{+}>k\right] ; e^{a X_{1}^{k}}\left|\psi\left(y+Y_{1}^{k}\right)\right|\right. \\
& \left.\cdot e^{a x} p^{*(n+1-k)}(d x d y d z)\right] \\
\leq & \frac{\|\psi\|_{\infty} C(a, \phi)}{k^{(N+3) / 2}} \\
& \cdot \mathbb{E}\left[\left[X_{k+1}^{n+1}<0\right] ; e^{a X_{k+1}^{n+1}}\right] \\
\leq & \frac{C_{2}}{k^{(N+3) / 2} \sqrt{n+1-k}} \cdot
\end{aligned}
$$

The assertion 3 ) follows since for any $\varepsilon>0$ one has

$$
\begin{aligned}
n^{(N+2) / 2} \sum_{k=l}^{n}\left|I_{n, k}\right| \leq & C_{1} \sum_{k=l}^{[n(1-\varepsilon)]} \frac{n^{(N+2) / 2}}{k^{3 / 2}(n+1-k)^{(N+2) / 2}} \\
& +C_{2} \sum_{[n(1-\varepsilon)+1]}^{n} \frac{n^{(N+2) / 2}}{k^{(N+3) / 2} \sqrt{n+1-k}} \\
\leq & \frac{C_{1}}{\varepsilon^{(N+2) / 2}} \sum_{k=l}^{[n(1-\varepsilon)]} \frac{1}{k^{3 / 2}} \\
& +\frac{C_{2}}{\sqrt{n}(1-\varepsilon)^{(N+3) / 2}} \sum_{[n(1-\varepsilon)+1]}^{n} \frac{1}{\sqrt{n+1-k}}
\end{aligned}
$$




$$
\leq C\left(\frac{1}{\varepsilon^{(N+2) / 2} \sqrt{l}}+\frac{\sqrt{\varepsilon}}{(1-\varepsilon)^{(N+3) / 2}}\right) .
$$

Since $\varepsilon$ is arbitrarily small, the assertion 3 ) follows.

The proof of ii) is also made by induction over $N$. If $g \in \mathcal{H}_{\delta}\left(\mathbb{R}^{N+1}\right)$ there exist $\phi \in \mathcal{H}_{\delta}\left(\mathbb{R}^{N}\right)$ and $\psi \in \mathcal{H}_{\delta}\left(\mathbb{R}^{1}\right)$ such that $|g| \leq \phi \otimes \psi$. We set

$$
I_{n}(y, z)=\mathbb{E}\left[\left[T_{+}>n\right] ; e^{a X_{1}^{n}} \phi\left(y+Y_{1}^{n}\right) \psi\left(z+Z_{1}^{n}\right)\right]
$$

and we have

$$
I_{n}(y, z)=\frac{1}{n+1} \sum_{k=0}^{n} I_{n, k}(y, z)
$$

with

$$
I_{n, k}(y, z)=\mathbb{E}\left[\left[T_{+}>k\right] \cap\left[X_{k+1}^{n+1}<0\right] ; e^{a X_{1}^{n+1}} \phi\left(y+Y_{1}^{n+1}\right) \psi\left(z+Z_{1}^{n+1}\right)\right]
$$

As above, one has

$$
\left|I_{n, k}(y, z)\right| \leq \inf \left\{\frac{C_{1}}{(n+1-k)^{(N+2) / 2} k^{3 / 2}}, \frac{C_{2}}{k^{(N+3) / 2} \sqrt{n+1-k}}\right\}
$$

which proves that the sequence

$$
\left\{n^{(N+2) / 2} \sum_{k=0}^{n}\left|I_{n, k}(y, z)\right|\right\}_{n \geq 1}
$$

is uniformly bounded in $y, z$. This achieves the proof of ii).

The convergence of the sequence

$$
\left\{n^{(N+3) / 2} \mathbb{E}\left[\left[T_{*+}>n\right] ; \varphi\left(X_{1}^{n}, Y_{1}^{n}\right)\right]\right\}_{n \geq 1}
$$

is obtained with similar arguments.

2.3. Behaviour of the process $\left(\left(X_{1}^{n}, \max \left\{0, X_{1}^{1}, \ldots, X_{1}^{n}\right\}, Y_{1}^{n}\right)\right)_{n \geq 0}$.

For any $n \geq 0$ set $\mathcal{X}_{1}^{n}=\max \left\{0, X_{1}^{1}, \ldots, X_{1}^{n}\right\}$ and let $T_{n}$ be the random variable defined on $(\Omega, \mathcal{F}, \mathbb{P})$ by $T_{n}=\inf \left\{0 \leq k \leq n: \mathcal{X}_{1}^{n}=\right.$ 
$\left.X_{1}^{k}\right\}$; for any continuous function $\varphi$ with compact support on $\mathbb{R}^{N+1}$ we have

$$
\begin{aligned}
& \mathbb{E}\left[\varphi\left(\mathcal{X}_{1}^{n}, \mathcal{X}_{1}^{n}-X_{1}^{n}, Y_{1}^{n}\right)\right] \\
& =\sum_{k=0}^{n} \mathbb{E}\left[\left[T_{n}=k\right] ; \varphi\left(X_{1}^{k},-X_{k+1}^{n}, Y_{1}^{n}\right)\right] \\
& =\sum_{k=0}^{n} \mathbb{E}\left[\left[0<X_{1}^{k}, X_{1}^{1}<X_{1}^{k}, \ldots, X_{1}^{k-1}<X_{1}^{k},\right.\right. \\
& \left.\left.\quad X_{1}^{k+1} \leq X_{1}^{k}, \ldots, X_{1}^{n} \leq X_{1}^{k}\right] ; \varphi\left(X_{1}^{k},-X_{k+1}^{n}, Y_{1}^{n}\right)\right] \\
& =\sum_{k=0}^{n} \mathbb{E}\left[\left[X_{1}^{1}>0, \ldots, X_{1}^{k}>0\right] \cap\left[X_{k+1}^{k+1} \leq 0, \ldots, X_{k+1}^{n} \leq 0\right] ;\right. \\
& \left.\varphi\left(X_{1}^{k},-X_{k+1}^{n}, Y_{1}^{n}\right)\right] .
\end{aligned}
$$

One obtains the following factorisation

$$
\mathbb{E}\left[\varphi\left(\mathcal{X}_{1}^{n}, \mathcal{X}_{1}^{n}-X_{1}^{n}, Y_{1}^{n}\right)\right]=\sum_{k=0}^{n} J_{n, k}(\varphi)
$$

with

$$
\begin{aligned}
& J_{n, k}(\varphi) \\
& \quad=\int_{\mathbb{R}^{N+1}} \varphi\left(x,-x^{\prime}, y+y^{\prime}\right) P_{-}^{k}((0,0), d x d y) P_{*+}^{n-k}\left((0,0), d x^{\prime} d y^{\prime}\right) .
\end{aligned}
$$

The behaviour of the process $\left(\mathcal{X}_{1}^{n}, \mathcal{X}_{1}^{n}-X_{1}^{n}, Y_{1}^{n}\right)$ is thus closely related to the one of the iterates of the transition kernels $P_{-}$and $P_{*+}$. Using this factorisation one proves the

Theorem 2.6. Suppose that the hypotheses of Theorem 2.2 hold.

Then, for any continuous function with compact support on $\mathbb{R}^{+} \times$ $\mathbb{R}^{+} \times \mathbb{R}^{N}$ the sequence

$$
\left\{n^{(N+3) / 2} \mathbb{E}\left[\varphi\left(\mathcal{X}_{1}^{n}, \mathcal{X}_{1}^{n}-X_{1}^{n}, Y_{1}^{n}\right)\right]\right\}_{n \geq 1}
$$


converges to

$$
\begin{aligned}
\frac{1}{(2 \pi)^{(N+1) / 2}} & \int_{\mathbb{R}^{+} \times \mathbb{R}^{+} \times \mathbb{R}^{N}} \varphi(s,-t, y) U^{*+}(d s) \lambda_{1}^{-} * U^{-}(d t) \lambda_{N}(d y) \\
+ & \frac{1}{(2 \pi)^{(N+1) / 2} \sqrt{|C|}} \\
& \cdot \int_{\mathbb{R}^{+} \times \mathbb{R}^{+} \times \mathbb{R}^{N}} \varphi(s,-t, y) \lambda_{+} * U^{*+}(d s) U^{-}(d t) \lambda_{N}(d y) .
\end{aligned}
$$

Furthermore, for any continuous function $f$ with compact support on $\mathbb{R}^{+} \times \mathbb{R}^{+}$and any $g$ in $\mathcal{H}_{\delta}\left(\mathbb{R}^{N}\right)$, the sequence

$$
\left\{n^{(N+3) / 2} \mathbb{E}\left[f\left(\mathcal{X}_{1}^{n}, \mathcal{X}_{1}^{n}-X_{1}^{n}\right) g\left(y+Y_{1}^{n}\right)\right]\right\}_{n \geq 1}
$$

is bounded, uniformly in $y \in \mathbb{R}^{N}$.

Proof. We only proof the first assertion; the second one may obtained with obvious modifications as in Theorem 2.5. Set $\varphi(x, t, y)=$ $\varphi_{1}(x) \varphi_{2}(t) \varphi_{3}(y)$ where $\varphi_{1}, \varphi_{2}$ and $\varphi_{3}$ are continuous with compact support. Fix $k \geq 0$; by Theorem 2.5 , the sequence

$$
\left\{n^{(N+3) / 2} \int_{\mathbb{R}^{-} \times \mathbb{R}^{N}} \varphi_{2}\left(x^{\prime}\right) \varphi_{3}\left(y+y^{\prime}\right) P_{*+}^{n-k}\left((0,0), d x^{\prime} d y^{\prime}\right)\right\}_{n \geq 1}
$$

is bounded uniformly in $y \in \mathbb{R}^{N}$ and converges to

$$
\frac{1}{(2 \pi)^{(N+1) / 2} \sqrt{|C|}} \int_{-\infty}^{0} \varphi_{2}(-t) \lambda_{1}^{-} * U^{-}(d t) \lambda_{N}\left(\varphi_{3}\right) .
$$

By the dominated convergence theorem, one thus obtains, for any fixed $i \geq 1$

$$
\begin{aligned}
& \lim _{n \rightarrow+\infty} n^{(N+3) / 2} \sum_{k=0}^{i} J_{n, k}(\varphi) \\
&=\frac{1}{(2 \pi)^{(N+1) / 2} \sqrt{|C|}} \sum_{k=0}^{i} \mathbb{E}\left[\left[T_{-}>k\right] ; \varphi_{1}\left(X_{1}^{k}\right)\right] \\
& \cdot \int_{-\infty}^{0} \varphi_{2}(-t) \lambda_{1}^{-} * U^{-}(d t) \lambda_{N}\left(\varphi_{3}\right) .
\end{aligned}
$$


In the same way one has

$$
\begin{aligned}
\lim _{n \rightarrow+\infty} n^{(N+3) / 2} & \sum_{k=n-i+1}^{n} J_{n, k}(\varphi) \\
= & \frac{1}{(2 \pi)^{(N+1) / 2} \sqrt{|C|}} \sum_{k=0}^{i} \mathbb{E}\left[\left[T_{*+}>k\right] ; \varphi_{2}\left(-X_{1}^{k}\right)\right] \\
& \cdot \lambda_{1}^{+} * U^{*+}\left(\varphi_{1}\right) \lambda_{N}\left(\varphi_{3}\right) .
\end{aligned}
$$

Note that the sums $\sum_{k=0}^{i} \mathbb{E}\left[\left[T_{-}>k\right] ; \varphi_{1}\left(X_{1}^{k}\right)\right]$ and $\sum_{k=0}^{i} \mathbb{E}\left[\left[T_{*+}>\right.\right.$ $\left.k] ; \varphi_{2}\left(X_{1}^{k}\right)\right]$ converges respectively to $U^{*+}\left(\varphi_{1}\right)$ and $\int_{-\infty}^{0} \varphi_{2}(-t) U^{-}(d t)$. To obtain the theorem it suffices to check that

$$
\limsup _{i \rightarrow+\infty} \limsup _{n \rightarrow+\infty}\left|n^{(N+3) / 2} \sum_{k=i+1}^{n-i} J_{n, k}(\varphi)\right|=0,
$$

one has

$$
\begin{aligned}
& \left|n^{(N+3) / 2} \sum_{k=i+1}^{[n / 2]} J_{n, k}(\varphi)\right| \\
& \leq n^{(N+3) / 2} \sum_{k=i+1}^{[n / 2]} \mathbb{E}\left[\left[T_{-}>k\right] ;\left|\varphi_{1}\left(X_{1}^{k}\right)\right|\right] \\
& \qquad \int_{\mathbb{R}^{-} \times \mathbb{R}^{N}}\left|\varphi_{2}\left(x^{\prime}\right)\right|\left|\varphi_{3}\left(y+y^{\prime}\right)\right| P_{*+}^{n-k}\left((0,0), d x^{\prime} d y^{\prime}\right) \\
& \leq C\left(\varphi_{2}, \varphi_{3}\right) \sum_{k=i+1}^{[n / 2]} \mathbb{E}\left[\left[T_{-}>k\right] ;\left|\varphi_{1}\left(X_{1}^{k}\right)\right|\right] \\
& \leq C(\varphi) \sum_{k=i+1}^{+\infty} \frac{1}{k^{3 / 2}} \cdot
\end{aligned}
$$

The same upperbound holds for the term

$$
n^{(N+3) / 2} \sum_{k=[n / 2]+1}^{n-i} J_{n, k}(\varphi) .
$$


This achieves the proof.

\section{A local limit theorem for a particular class of solvable groups.}

Recall that $G=G_{d, N}=\mathbb{R}^{*+} \times \mathbb{R}^{d} \times \mathbb{R}^{N}$ with the composition law

$$
g \cdot g^{\prime}=\left(a a^{\prime}, a u^{\prime}+u, b+b^{\prime}\right),
$$

for all $g=(a, u, b)$, for all $g^{\prime}=\left(a^{\prime}, u^{\prime}, b^{\prime}\right) \in G_{d, N}$.

The proof of Theorem 1.1 is closed to the one of the local limit theorem for the affine group of the real line given in [7]; we just give here the main steps of the demonstration.

Let us first introduce some helpfull notations. Let $g_{n}=\left(a_{n}, u_{n}, b_{n}\right)$, $n=1,2, \ldots$ be independent and identically distributed random variables with distribution $\mu$. Denote by $\mathcal{F}_{n}$ the $\sigma$-algebra generated by the variables $g_{1}, g_{2}, \ldots, g_{n}, n \geq 1$. For any $n \geq 1$, set $G_{1}^{n}=g_{1} \cdots g_{n}=$ $\left(A_{1}^{n}, U_{1}^{n}, B_{1}^{n}\right)$; we have $A_{1}^{n}=a_{1} \cdots a_{n}, U_{1}^{n}=\sum_{k=1}^{n} a_{1} \cdots a_{k-1} u_{k}$ and $B_{1}^{n}=b_{1}+\cdots+b_{n}$. More generally, if $1 \leq m \leq n$, set $A_{m}^{n}=a_{m} \cdots a_{n}$, $U_{m}^{n}=\sum_{k=m}^{n} a_{m} \cdots a_{k-1} u_{k}, B_{m}^{n}=b_{m}+\cdots+b_{n}$ and set $A_{m}^{n}=1$, $U_{m}^{n}=0, B_{m}^{n}=0$ otherwise.

Let $\tilde{\mu}$ be the image of $\mu$ by the map

$$
g=(a, u, b) \longmapsto \tilde{g}=\left(\frac{1}{a}, \frac{u}{a}, b\right),
$$

if $\tilde{g}_{n}=\left(\tilde{a}_{n}, \tilde{u}_{n}, \tilde{b}_{n}\right), n=1,2, \ldots$ are independent and identically distributed random variables with distribution $\tilde{\mu}$ on $G$, set $\tilde{G}_{m}^{n}=\tilde{g}_{m} \cdots \tilde{g}_{n}$ $=\left(\tilde{A}_{m}^{n}, \tilde{U}_{m}^{n}, \tilde{B}_{m}^{n}\right)$.

In order to obtain the asymptotic behaviour of the power of convolution $\mu^{* n}$ we use the fact that the sequence $\left\{U_{1}^{n}\right\}_{n>1}$ behaves like the maximum of the variables $A_{1}^{1}, \ldots, A_{1}^{n}$. These idea was already used in [7]. Set $\mathcal{A}=\{g=(a, u, b) \in G: a>1\}$ and consider the transition kernel $P_{\mathcal{A}}$ associated with $(\mu, \mathcal{A})$ and defined by

$$
P_{\mathcal{A}}(g, \mathcal{B})=\int_{G} \mathbf{1}_{\mathcal{A}^{c} \cap \mathcal{B}}(g h) \mu(d h)
$$

for any Borel set $\mathcal{B} \subset G$ and any $g \in G$. The probabilistic interpretation of $P_{\mathcal{A}}$ is the following one: if $T_{\mathcal{A}}=\inf \left\{n \geq 1: G_{1}^{n} \in \mathcal{A}\right\}$ is the first entrance time in $\mathcal{A}$ of the random walk $\left\{G_{1}^{n}\right\}_{n \geq 0}$ then

$$
P_{\mathcal{A}}^{n}(e, \mathcal{B})=\mathbb{P}\left[\left[T_{\mathcal{A}}>n\right] \cap\left[G_{1}^{n} \in \mathcal{B}\right]\right], \quad \text { for all } n \geq 1 \text {. }
$$


In the same way, set $\mathcal{A}^{\prime}=\{g \in G: a(g) \geq 1\}$, let $\tilde{P}_{\mathcal{A}^{\prime}}$ be the operator associated with $\left(\tilde{\mu}, \mathcal{A}^{\prime}\right)$ and denote by $\tilde{T}_{\mathcal{A}^{\prime}}$ the first entrance time in $\mathcal{A}^{\prime}$ of the random walk $\left\{\tilde{G}_{1}^{n}\right\}_{n \geq 1}$; one has

$$
\tilde{P}_{\mathcal{A}^{\prime}}^{n}(e, \mathcal{B})=\mathbb{P}\left[\left[\tilde{T}_{\mathcal{A}^{\prime}}>n\right] \cap\left[\tilde{G}_{1}^{n} \in \mathcal{B}\right]\right], \quad \text { for all } n \geq 1 .
$$

As in Section 2.3, we introduce the first time at which the random walk $\left\{A_{1}^{n}\right\}_{n \geq 1}$ reaches its maximun on $\mathbb{R}^{*+}$; for any continuous function $\varphi$ with compact support on $G$, we thus obtain

$$
\mathbb{E}\left[\varphi\left(G_{1}^{n}\right)\right]=\sum_{k=0}^{n} I_{n, k}(\varphi),
$$

where

$I_{n, k}(\varphi)=\int_{G \times G} \varphi\left(\frac{a^{\prime}}{a}, \frac{u+u^{\prime}}{a}, b+b^{\prime}\right) \tilde{P}_{\mathcal{A}^{\prime}}^{k}(e, d a d u d b) P_{\mathcal{A}}^{n-k}\left(e, d a^{\prime} d u^{\prime} d b^{\prime}\right)$.

We now give the main steps of the proof of Theorem 1.1 under hypothesis G1, G2 and G3.

First step. Control of the central terms of the sum $\sum_{k=0}^{n} I_{n, k}(\varphi)$.

We show here that

$$
\limsup _{i \rightarrow+\infty} \limsup _{n \rightarrow+\infty} \sum_{k=i}^{n-i} I_{n, k}(\varphi)=0 .
$$

Without loss of generality, one may suppose that the support of $\varphi$ is included in $\mathbb{R}^{*+} \times\left(\mathbb{R}^{*+}\right)^{d} \times \mathbb{R}^{N}$; for any $\varepsilon>0$ there exist a constant $C>0$ and a positive function $\phi$ with compact support on $\mathbb{R}^{N}$ such that

$$
\varphi(a, u, b) \leq C \frac{a^{\varepsilon}}{\|u\|^{2 \varepsilon}} \phi(b),
$$

it follows that for any $(\alpha, \beta)$ in $\mathbb{R}^{*+} \times \mathbb{R}^{N}$

$$
\begin{aligned}
& \mathbb{E}\left[\left[T_{\mathcal{A}}>l\right] ; \varphi\left(\frac{A_{1}^{l}}{\alpha}, \frac{u+U_{1}^{l}}{\alpha}, \beta+B_{1}^{l}\right)\right] \\
& \leq C \alpha^{\varepsilon} \mathbb{E}\left[\left[a_{1} \leq 1\right] \cap\left[\max \left\{A_{2}^{2}, \ldots, A_{2}^{l}\right\} \leq \frac{1}{a_{1}}\right] ; \frac{\left(A_{1}^{l}\right)^{\varepsilon}}{\left\|u+U_{1}^{l}\right\|^{2 \varepsilon}} \phi\left(\beta+B_{1}^{l}\right)\right] \\
& \leq C \alpha^{\varepsilon} \int_{G} \mathbb{E}\left[\frac{\left(A_{2}^{l}\right)^{\varepsilon}}{\max \left\{A_{2}^{2}, \ldots, A_{2}^{l}\right\}^{2 \varepsilon}} \phi\left(\beta+b+B_{2}^{l}\right)\right] \frac{\mu(d a d v d b)}{a^{\varepsilon}\|v\|^{2 \varepsilon}}
\end{aligned}
$$


the last inequality being a consequence of the fact that $\left\|u+U_{1}^{l}\right\| \geq\left\|u_{1}\right\|$ $\mathbb{P}$-almost surely and

$$
\mathbf{1}_{\left\{\max \left\{A_{2}^{2}, \ldots, A_{2}^{l}\right\} \leq 1 / a_{1}\right\}} \leq \frac{1}{a_{1}^{2 \varepsilon} \max \left\{A_{2}^{2}, \ldots, A_{2}^{l}\right\}^{2 \varepsilon}} .
$$

By Theorem 2.6 one obtains

$$
l^{(N+3) / 2} \mathbb{E}\left[\left[T_{\mathcal{A}}>l\right] ; \varphi\left(\frac{A_{1}^{l}}{\alpha}, \frac{u+U_{1}^{l}}{\alpha}, \beta+B_{1}^{l}\right)\right] \leq C_{1}(\varphi) \alpha^{\varepsilon} .
$$

The same upperbound holds under hypotheses G1, G2 and G'3 (see [7, Lemma 3.1]).

It readily follows that

$$
\begin{aligned}
n^{(N+3) / 2} \sum_{k=i}^{[n / 2]} I_{n, k}(\varphi) & \leq 2^{(N+3) / 2} \sum_{k=i}^{[n / 2]}(n-k)^{(N+3) / 2} I_{n, k}(\varphi) \\
& \leq C_{1}(\varphi) \sum_{k=i}^{[n / 2]} \mathbb{E}\left[\left[\tilde{T}_{\mathcal{A}^{\prime}}>k\right] ;\left(\tilde{A}_{1}^{k}\right)^{\varepsilon}\right] \\
& \leq C_{2}(\varphi) \sum_{k=i}^{[n / 2]} \frac{1}{k^{3 / 2}}
\end{aligned}
$$

and so

$$
\limsup _{i \rightarrow+\infty} \limsup _{n \rightarrow+\infty} n^{(N+3) / 2} \sum_{k=i}^{[n / 2]} I_{n, k}(\varphi)=0 .
$$

The control of the sum $\sum_{k=[n / 2]}^{n-i} I_{n, k}(\varphi)$ goes along the same lines.

Second step. Convergence of the sequence

$$
l^{(N+3) / 2} \mathbb{E}\left[\left[T_{\mathcal{A}}>l\right] ; \varphi\left(\frac{A_{1}^{l}}{\alpha}, \frac{u+U_{1}^{l}}{\alpha}, \beta+B_{1}^{l}\right)\right]
$$

for any $(\alpha, u, \beta) \in] 0,1] \times\left(\mathbb{R}^{*+}\right)^{d} \times \mathbb{R}^{N}$.

It is the more technical part of the proof and it uses and idea due to Afanasev [1]. Without loss of generality, one may suppose $\alpha=1$, $u=0$ and $\beta=0$. For any $n \geq 1$, set

$$
\mathbb{E}_{n}(\varphi)=n^{(N+3) / 2} \mathbb{E}\left[\left[T_{A}>n\right] ; \varphi\left(A_{1}^{n}, U_{1}^{n}, B_{1}^{n}\right)\right] .
$$


Fix $i \in \mathbb{N}$ such that $1 \leq i \leq n / 2$ and consider

$$
\mathbb{E}_{n}(\varphi, i)=n^{(N+3) / 2} \mathbb{E}\left[\left[T_{A}>n\right] ; \varphi\left(A_{1}^{n}, U_{1}^{i}+A_{1}^{n-i} U_{n-i+1}^{n}, B_{1}^{n}\right)\right] .
$$

To obtain the claim, it suffices to prove that

a) $\limsup \limsup \left|\mathbb{E}_{n}(\varphi)-\mathbb{E}_{n}(\varphi, i)\right|=0$,

b) for any fixed $n \in \mathbb{N}$, the sequence $\left\{\mathbb{E}_{n}(\varphi, i)\right\}_{n \geq 1}$ converges to a finite limit.

Proof of CONVERGEnCE a). We use the equality

$$
U_{1}^{n}=U_{1}^{i}+A_{1}^{i} U_{i+1}^{n-i}+A_{1}^{n-i} U_{n-i+1}^{n},
$$

without loss of generality one may suppose that $\varphi$ is continuously differentiable, and so, for any $\varepsilon>0$ there exists $C>0$ and a positive function $\phi$ with compact support on $\mathbb{R}^{N}$ such that

$$
|\varphi(a, u, b)-\varphi(a, v, b)| \leq C a^{\varepsilon}\|u-v\|^{\varepsilon} \phi(b),
$$

consequently

$$
\begin{aligned}
\mid \mathbb{E}_{n}(\varphi) & -\mathbb{E}_{n}(\varphi, i) \mid \\
& \leq C n^{(N+3) / 2} \mathbb{E}\left[\left[T_{A}>n\right] ;\left(A_{1}^{n}\right)^{\varepsilon}\left(A_{1}^{i}\right)^{\varepsilon}\left\|U_{i+1}^{n-i}\right\|^{\varepsilon} \phi\left(B_{1}^{n}\right)\right] \\
& \leq C n^{(N+3) / 2} \sum_{k=i+1}^{n-i} \mathbb{E}\left[\left[T_{A}>n\right] ;\left(A_{1}^{n}\right)^{\varepsilon}\left(A_{1}^{k-1}\right)^{\varepsilon}\left\|u_{k}\right\|^{\varepsilon} \phi\left(B_{1}^{n}\right)\right] .
\end{aligned}
$$

Note that for $i \leq k \leq[n / 2]$ one has

$$
\begin{aligned}
& \mathbb{E}\left[\left[T_{A}>n\right] ;\left(A_{1}^{n}\right)^{\varepsilon}\left(A_{1}^{k-1}\right)^{\varepsilon}\left\|u_{k}\right\|^{\varepsilon} \phi\left(B_{1}^{n}\right)\right] \\
& \leq \mathbb{E}\left[\left[T_{A}>k-1\right] \cap\left[\max \left\{A_{k+1}^{k+1}, \ldots, A_{k+1}^{n}\right\} \leq \frac{1}{A_{1}^{k}}\right] ;\right. \\
& \left.\quad\left(A_{1}^{n}\right)^{\varepsilon}\left(A_{1}^{k-1}\right)^{\varepsilon}\left\|u_{k}\right\|^{\varepsilon} \phi\left(B_{1}^{n}\right)\right] \\
& \leq \mathbb{E}\left[\left[T_{A}>k-1\right] ;\left(A_{1}^{k-1}\right)^{\varepsilon / 2} a_{k}^{-\varepsilon / 2}\left\|u_{k}\right\|^{\varepsilon}\right. \\
& \left.\cdot \max \left\{A_{k+1}^{k+1}, \ldots, A_{k+1}^{n}\right\}^{-3 \varepsilon / 2}\left(A_{k+1}^{n}\right)^{\varepsilon} \phi\left(B_{1}^{n}\right)\right] .
\end{aligned}
$$

By Theorem 2.6,

$$
(n-k)^{(N+3) / 2} \mathbb{E}\left[\max \left\{A_{k+1}^{k+1}, \ldots, A_{k+1}^{n}\right\}^{-3 \varepsilon / 2}\left(A_{k+1}^{n}\right)^{\varepsilon} \phi\left(\beta+B_{k+1}^{n}\right)\right]
$$


is bounded, uniformly in $\beta \in \mathbb{R}^{N}$ and so

$$
(n-k)^{(N+3) / 2} E\left[\left[T_{A}>n\right] ;\left(A_{1}^{n}\right)^{\varepsilon}\left(A_{1}^{k-1}\right)^{\varepsilon}\left\|u_{k}\right\|^{\varepsilon} \phi\left(B_{1}^{n}\right)\right] \leq \frac{C_{1}}{k^{3 / 2}} .
$$

When $[n / 2] \leq k \leq n-i$ one obtains by a similar argument

$$
k^{(N+3) / 2} \mathbb{E}\left[\left[T_{A}>n\right] ;\left(A_{1}^{n}\right)^{\varepsilon}\left(A_{1}^{k-1}\right)^{\varepsilon}\left\|u_{k}\right\|^{\varepsilon} \phi\left(B_{1}^{n}\right)\right] \leq \frac{C_{2}}{(n-k)^{3 / 2}} .
$$

Finally one has

$$
\left|\mathbb{E}_{n}(\varphi)-\mathbb{E}_{n}(\varphi, i)\right| \leq C_{3} \frac{1}{\sqrt{i}}
$$

convergence a) follows.

Proof of COnvergence b). Fix an integer $i$; we have

$$
\begin{aligned}
& \mathbb{E}_{n}(\varphi, i) \\
& \quad=\int_{G} E_{n}\left(\varphi, g, h_{1}, h_{2}, \ldots, h_{i}\right) P_{\mathcal{A}}^{i}(e, d g) \mu\left(d h_{1}\right) \mu\left(d h_{2}\right) \cdots \mu\left(d h_{i}\right)
\end{aligned}
$$

with

$$
\begin{aligned}
& E_{n}\left(\varphi, g, h_{1}, h_{2}, \ldots, h_{i}\right) \\
& =\mathbb{E}\left[\left[\max \left\{A_{i+1}^{i+1}, \ldots, A_{i+1}^{n-i}\right\} \leq \frac{1}{a(g)}\right]\right. \\
& \cap\left[A_{i+1}^{n-i} \leq \min \left\{\frac{1}{a(g)}, \frac{1}{a(g) a\left(h_{1}\right)}, \ldots, \frac{1}{a(g) a\left(h_{1}\right) \cdots a\left(h_{i}\right)}\right\}\right] ; \\
& \\
& \varphi\left(a(g) A_{i+1}^{n-i} a\left(h_{1}\right) \cdots a\left(h_{i}\right), u(g)+a(g) A_{i+1}^{n-i} u\left(h_{1} \cdots h_{i}\right),\right. \\
& \left.B_{1}^{n-i}+b\left(h_{1}\right)+\cdots+b\left(h_{i}\right)\right] .
\end{aligned}
$$

Using Theorem 2.6, one may see that, for any $g, h_{1}, \ldots, h_{i} \in G$, the sequence

$$
\left\{n^{(N+3) / 2} E_{n}\left(\varphi, g, h_{1}, h_{2}, \cdots, h_{i}\right)\right\}_{n \geq 1}
$$

converges to a finite limit. To obtain the convergence $b$ ), we have to use Lebesgue dominated convergence theorem and therefore, we have to obtain an appropriate upperbound for $n^{(N+3) / 2} E_{n}\left(\varphi, g, h_{1}, h_{2}, \ldots, h_{i}\right)$. 
Using the fact that for any $\varepsilon>0$ there exist $C>0$ and a positive continuous function $\phi$ with compact support on $\mathbb{R}^{N}$ such that $|\varphi(a, u, b)| \leq C a^{\varepsilon} \phi(b)$, one thus obtains

$$
n^{(N+3) / 2} E_{n}\left(\varphi, g, h_{1}, h_{2}, \ldots, h_{i}\right) \leq C_{1} a(g)^{-3 \varepsilon / 2} a\left(h_{1}\right)^{\varepsilon} \cdots a\left(h_{i}\right)^{\varepsilon}
$$

which allows us to use the Lebesgue dominated convergence theorem for $\varepsilon$ small enough; convergence b) follows.

Consequently, $\left\{n^{(N+3) / 2} I_{n, 0}(\varphi)\right\}_{n \geq 1}$ converges to a finite limit; furthermore, for any $i \geq 1$ and any compact set $K \subset \mathbb{R}^{*+} \times \mathbb{R}^{N}$, the dominated convergence theorem ensures the existence of a finite limit as $n$ goes to $+\infty$ for

$$
\left\{n^{(N+3) / 2} \sum_{k=0}^{i} I_{n, k}(\varphi, K)\right\}_{n \geq 1},
$$

where

$$
\begin{aligned}
I_{n, k}(\varphi, K)= & \int_{G} \mathbf{1}_{K}(g) \\
& \cdot\left(\int_{G} \varphi\left(\frac{a(h)}{a(g)}, \frac{u(g)+u(h)}{a(g)}, b(g)+b(h)\right) P_{\mathcal{A}}^{n-k}(e, d h)\right) \\
& \cdot \tilde{P}_{\mathcal{A}^{\prime}}^{k}(e, d g) .
\end{aligned}
$$

The following step shows that the indicator function $\mathbf{1}_{K}$ does not disturb too much the behaviour of theses integrals.

Third step. Control of the residual terms.

In the first step of the present proof, we have shown that, for any $\varepsilon>0$ there exists $C_{1}>0$ such that

$$
\begin{aligned}
(n-k)^{(N+3) / 2} \mathbb{E}\left[\left[T_{\mathcal{A}}>n-k\right] ; \varphi\left(\frac{A_{1}^{n-k}}{\alpha}, \frac{u+U_{1}^{n-k}}{\alpha}, \beta+B_{1}^{n-k}\right)\right] \\
\leq C_{1}(\varphi) \alpha^{\varepsilon} .
\end{aligned}
$$

It follows that for any $0<\delta<1$

$$
\sum_{k=1}^{i} \int_{\{g \in G: a(g) \leq \delta\}}\left(\int_{G} \varphi\left(\frac{a(h)}{a(g)}, \frac{u(g)+u(h)}{a(g)}, b(g)+b(h)\right) P_{\mathcal{A}}^{n-k}(e, d h)\right)
$$




$$
\begin{aligned}
& \cdot \tilde{P}_{\mathcal{A}^{\prime}}^{k}(e, d g) \\
& \quad \leq C_{1} \sum_{k=1}^{i} \frac{1}{(n-k)^{(N+3) / 2}} \mathbb{E}\left[\left[\tilde{T}_{\mathcal{A}^{\prime}}>k\right] ;\left(\tilde{A}_{1}^{k}\right)^{\varepsilon}\right] \\
& \quad \leq C_{1} \sum_{k=1}^{i} \frac{1}{(n-k)^{(N+3) / 2} k^{3 / 2}} .
\end{aligned}
$$

On the other hand for any fixed $U>0$, one has

$$
\begin{gathered}
\sum_{k=1}^{i} \int_{\{g \in G:\|u(g)\| \geq U\}}\left(\int_{G} \varphi\left(\frac{a(h)}{a(g)}, \frac{u(g)+u(h)}{a(g)}, b(h)+b(g)\right) P_{\mathcal{A}}^{n-k}(e, d h)\right) \\
\cdot \tilde{P}_{\mathcal{A}^{\prime}}^{k}(e, d g) \\
\leq \frac{C_{1}}{U^{\varepsilon / 2}} \sum_{k=1}^{i} \frac{1}{(n-k)^{(N+3) / 2}} E\left[\left[\tilde{T}_{\mathcal{A}^{\prime}}>k\right] ;\left(\tilde{A}_{1}^{k}\right)^{\varepsilon}\left\|\tilde{U}_{k}\right\|^{\varepsilon / 2}\right] \\
\leq \frac{C_{1}}{U^{\varepsilon / 2}} \sum_{k=1}^{i} \frac{1}{(n-k)^{(N+3) / 2} k^{3 / 2}} .
\end{gathered}
$$

The last inequality being guaranteed by standart estimations. (see [7, Lemma 3.3] for more details).

\section{References.}

[1] Afanas'ev, V. I., On a maximum of a transient random walk in random environment. Theory Probab. Appl. 35 (1987), 205-215.

[2] Billingsley, P., Ergodic theory and information. Wiley Series in Probability and Mathematical statistics, 1964.

[3] Bougerol, Ph., Exemples de théorèmes locaux sur les groupes résolubles. Ann. Inst. H. Poincaré 19 (1983), 369-391.

[4] Breiman, L., Probability. Addison-Wesley Publishing Company, 1964.

[5] Grincevicius, A. K., A central limit theorem for the group of linear transformation of the real axis. Soviet Math. Doklady 15 (1974), 15121515 .

[6] Iglehart, D. L., Random walks with negative drift conditioned to stay positive. J. Appl. Probab. 11 (1974), 742-751.

[7] Le Page, E., Peigné, M., A local limit theorem on the semi-direct product of $\mathbb{R}^{*+}$ and $\mathbb{R}^{d}$. Ann. Inst. H. Poincaré 2 (1997), 223-252. 
[8] Spitzer, F., Principles of random walks. D. Van Nostrand Company, 1964.

[9] Sunyach, Ch., Sur les fluctuations des marches aléatoires sur un groupe. Lecture Notes in Math. 1096 (1984), 549-558.

[10] Varopoulos, N. Th., Wiener-Hopf theory and nonunimodular groups. $J$. Funct. Anal. 120 (1994), 467-483.

[11] Varopoulos, N. Th., Analysis on Lie groups. Revista Mat. Iberoamaricana 12 (1996), 791-917.

Recibido: 29 de octubre de 1.997

Emile Le Page

Institut Mathématique de Rennes

Université de Bretagne Sud

1 Rue de la Loi

Vannes 56000, FRANCE

emile.lepage@univ-ubs.fr

and

Marc Peigné

Institut Mathématique de Rennes

Université de Rennes I

Campus de Beaulieu

35042 Rennes Cedex, FRANCE

peigne@univ-rennes1.fr 